\title{
A Rare Catastrophe: Three Cases of Aortic Root Dehiscence after Surgery
}

\author{
Taner İyigün'1, MD; Barış Timur' ${ }^{1}$, MD; Timuçin Aksu' ${ }^{1}$, MD
}

DOI: 10.21470/1678-9741-2020-0679

\begin{abstract}
Ascending aortic pathologies may be life-threatening. Postoperative aortic root dehiscence is a very rare but extremely dangerous complication with a high mortality rate, and redo surgery is mandatory due to high risk of spontaneous rupture. We present three cases that had undergone Bentall procedure and had postoperative aortic root dehiscence. One of the patients presented with hemiplegia caused by septic embolus while
\end{abstract}

the others had mild symptoms. Dr. Yakut's modified Bentall procedure, the flanged technique, was performed for each patient in redo surgery. Two patients were successfully discharged from the hospital, but one died due to intracranial hemorrhage and multiple organ failure.

Keywords: Aortic Diseases. Ascending Aorta. Dehiscence. Cardiac Surgery. Rupture. Spontaneous.

\begin{tabular}{ll}
\hline Abbreviations, acronyms \& symbols \\
\hline CABG $\quad=$ Coronary artery bypass grafting \\
CT $\quad=$ Computed tomography \\
ICU $\quad=$ Intensive care unit \\
\hline
\end{tabular}

\section{INTRODUCTION}

Ascending aortic pathologies may be life-threatening due to rupture or dissection. Acute untreated aortic dissection has a mortality rate up to $50 \%$ within 48 hours. Emergency surgery statistically significantly decreases the mortality rate. Elective cases have lower (3-5\%) mortality rates compared with emergency cases $(15-26 \%)^{[1]}$. Bentall and De Bono first described the procedure for ascending aortic pathologies - that include aortic valve and sinuses of Valsalva pathologies - in $1968^{[2]}$. Bentall-De Bono procedure is an effective way to treat dilated ascending aorta with concomitant aortic root and aortic valve pathologies. Aortic root dehiscence and pseudoaneurysm formation following a surgery is a rare but extremely dangerous complication. The most common cause for graft dehiscence in these patients is infection, while surgical technical errors

'Department of Cardiovascular Surgery, Istanbul Mehmet Akif Ersoy Thoracic and Cardiovascular Surgery Research and Training Hospital, Istanbul, Turkey.

This study was carried out at the Department of Cardiovascular Surgery, istanbul Mehmet Akif Ersoy Thoracic and Cardiovascular Surgery Research and Training Hospital, Istanbul, Turkey. and recurrent aneurysmal dilatation are the other causes ${ }^{[3]}$. Most common sites of dehiscence were the distal suture line, proximal valvular sutures, and coronary button anastomosis sites ${ }^{[4]}$. Mortality rate for the patients complicated by aortic root dehiscence remains noticeably high even if they had the chance of emergency redo surgery ${ }^{[5]}$. Here we present three cases of postoperative aortic root dehiscence.

\section{Case 1}

A 51-year-old male patient with diagnosis of type 1 aortic dissection had rheumatoid arthritis. Echocardiography revealed dissection flap with moderate aortic valve insufficiency. Contrasted computed tomography (CT) proved the diagnosis of acute type 1 aortic dissection. He was successfully discharged at postoperative $12^{\text {th }}$ day of Bentall procedure. Six months later, the patient was admitted to the emergency department suffering from dyspnea. Contrasted CT revealed type B dissection with periaortic hematoma. He was followed with medical therapy as he refused the surgery, but, unfortunately, he was transferred to the intensive care unit (ICU) in the fourth day of hospitalisation and undergone surgery. Modified Bentall procedure, the flanged technique, with mechanical aortic conduit was used

Correspondence Address:

\section{Barış Timur}

(D) https://orcid.org/0000-0003-4446-6374

Mehmet Akif Ersoy Gogus Kalp Damar Cerrahisi Egitim Arastirma Hastanesi, Kalp Damar Cerrahisi Bölümü, Bezirganbahce mevki, Kucukcekmece, Istanbul, Turkey Zip Code: 34303

E-mail:dr.baristimur@gmail.com 
for the patient whose aortic root was dehiscent by the proximal valvular sutures ${ }^{[6]}$ (Figure 1). Aortic root was also strengthened using bovine pericardium. Postoperative period was complicated by subdural hematoma, and the patient died four months after the surgery with multiorgan failure.

\section{Case 2}

A 45-year-old male patient with Marfanoid stature was admitted to our clinic with occasional chest and back pain. Contrasted $\mathrm{CT}$ and transthoracic echocardiography were performed. The ascending aorta and sinuses of Valsalva were

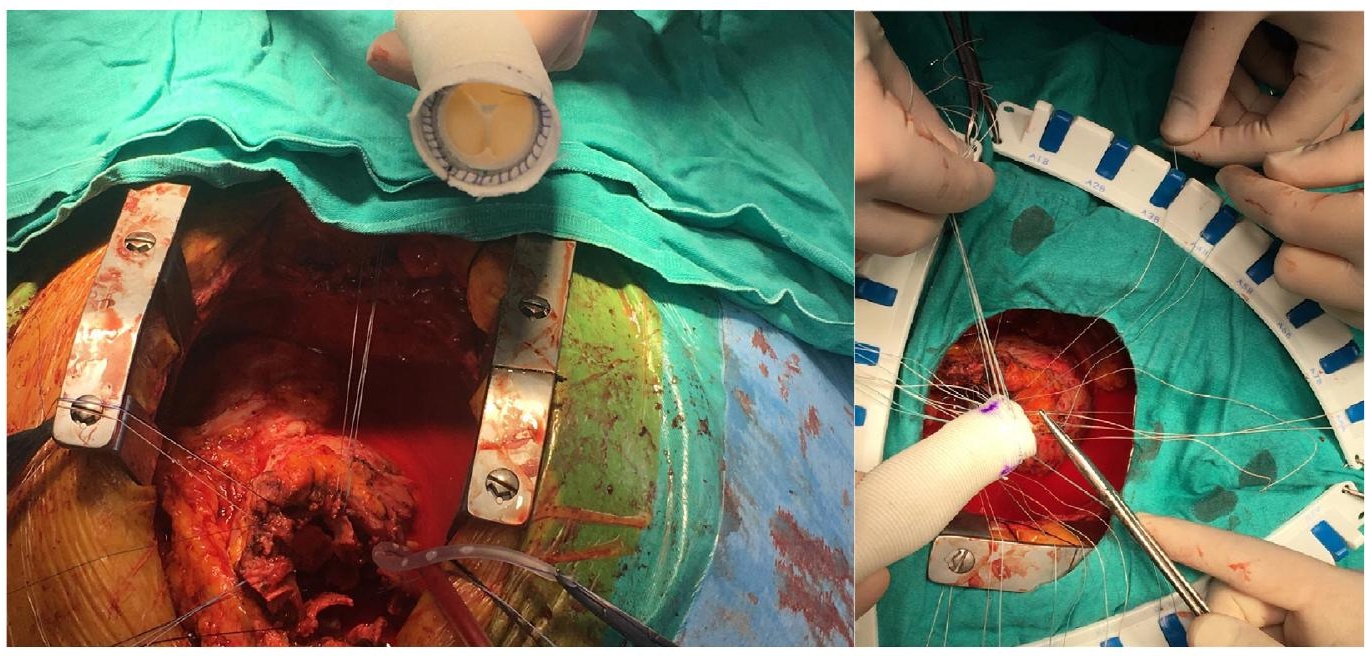

Fig. 1 - Preparation of Dr. Yakut's flanged Bentall graft. $51 \mathrm{~mm}$ and $59 \mathrm{~mm}$ in diameter, respectively, with moderate aortic

stenosis. After emergency preoperative follow-up, the patient underwent Bentall procedure. On the $7^{\text {th }}$ day, he was discharged from the hospital without any complications. Two weeks after the surgery, the patient was admitted to the hospital with fever, dyspnea, and fatigue. Blood count and biochemical markers were within normal ranges, there was nothing remarkable in urine and blood cultures, and echocardiography was normal. At the $10^{\text {th }}$ day of admission, with prophylactic antibiotic therapy, his general medical condition deteriorated, which was accompanied by tachycardia and hypotension. Leak from aortic root was diagnosed by transesophageal echocardiography, and hematoma around ascending aorta was shown by contrasted thorax CT (Figure 2A). Emergency redo Bentall procedure, flanged technique, with mechanical aortic conduit was performed ${ }^{[6]}$. Aortic root was strengthened using bovine pericardium. The patient was discharged at the postoperative $19^{\text {th }}$ day without any other complications.

\section{Case 3}

A 69-year-old male patient with previous diagnosis of diabetes mellitus and hypertension suffering from angina provoked by physical exertion was hospitalised with the diagnosis of ascending aortic aneurysm and severe aortic regurgitation revealed by contrasted $\mathrm{CT}$ and transthoracic echocardiography. Atherosclerotic coronary artery disease was also diagnosed during preoperative follow-up. Coronary artery bypass grafting (CABG) (left anterior descending artery, obtuse marginal first and second branches of circumflex artery, and right coronary artery) and Bentall procedure with mechanical aortic conduit were performed. He was extubated seven hours after the surgery, discharged from ICU on the postoperative $1^{\text {st }}$ day, and discharged from the hospital on the postoperative $7^{\text {th }}$ day. Four months after surgery, in another hospital, infective endocarditis of mechanical valve was diagnosed after his admission with left sided hemiplegia caused by septic emboli. The patient was transferred to our hospital and after four days due to increasing thrombocyte count (from 47000 to 90000), and redo CABG and flanged Bentall technique with bioprosthetic aortic conduit were performed ${ }^{[6]}$. Aortic root was strengthened using bovine pericardium. Vegetations were seen on the leaflet (Figure 2B). He was discharged from the hospital on the postoperative $45^{\text {th }}$ day after termination of antibiotic therapy and referred to physical therapy and rehabilitation for hemiplegia.

\section{DISCUSSION}

Aortic root dehiscence is a rare phenomenon. There are some limited series and case reports in the literature regarding pseudoaneurysm of thoracic

Fig. 2 - A, B) Preoperative images of the patients' dehiscenced aortic root. 
aorta ${ }^{[3,4,7-11]}$. Due to its insidious onset, diagnosis of the pathology can be challenging. Clinical presentation of aortic root dehiscence may present with various symptoms. Different series and cases on the matter showed numerous symptoms such as heart failure, chest pain, syncope, headache (due to intracranial infarction), fever, dyspnea, and even seizures $s^{[3,7,9-11]}$. Symptoms such as heart failure and chest pain may lead the physician to further investigate the aorta and heart. However, nonspecific symptoms such as fever or symptoms of the central nervous system may mislead the physician. It should be kept in mind that patients that had undergone ascending aortic and aortic root surgery should be investigated thoroughly, no matter the symptoms he/she has.

The underlying pathology that causes aortic pseudoaneurysm may vary as well. The most encountered reason was infection of various anatomic structures such as valve, aortic graft, or mediastinum ${ }^{[3,4,7-10]}$. Different types of microorganisms were reported to be isolated from the cultures, such as Staphylococcus aureus, Enterococcus, and Candida ${ }^{[3,8-10]}$. On the other hand, Gebhard et al. ${ }^{[1]]}$ presented a case with high-dose corticosteroid use after the surgery. As a concomitant risk factor, using a prosthetic valved conduit increases the tendency for dehiscence of the aortic root. Moreover, predisposing factors like rheumatoid arthritis, history of prolonged corticosteroid use, and connective tissue disorder may complicate the postoperative period of successfully managed aortic root surgeries.

The time interval and the reason of dehiscence after surgery may differ. There are cases reported up to 17 years after the first operation ${ }^{[12]}$. Infectious and non-infectious causes were present for dehiscence to occur ${ }^{[7]}$.

Brewer et al. ${ }^{[13]}$, Thubrikar et al. ${ }^{[14]}$, and Lansac et al. ${ }^{[15]}$ proved the importance of the dynamic properties of aortic root. Therefore, using a mechanical conduit may disrupt this dynamic feature of the aortic root and ascending aorta and lead to dehiscence. Yakut's modified surgical technique leading to postsurgical aortic root most similar to physiological root may be the reason of surgical success $s^{[6]}$.

\section{CONCLUSION}

Aortic root dehiscence after aortic root surgeries is an extremely rare but mortal complication. Redo surgery is mandatory due to high risk of spontaneous rupture. We prefer and advise the use of flanged Bentall technique for reoperations after root dehiscence, as it provides physiologic motion of the root more than other modified Bentall procedures. The flanged technique may be an effective alternative method for aortic root dehiscence after surgery.

\section{No financial support. \\ No conflict of interest.}

\section{Authors' roles \& responsibilities}

Ti Substantial contributions to the conception or design of the work; or the acquisition, analysis, or interpretation of data for the work; final approval of the version to be published

BT

Drafting the work or revising it critically for important intellectual content; agreement to be accountable for all aspects of the work in ensuring that questions related to the accuracy or integrity of any part of the work are appropriately investigated and resolved; final approval of the version to be published

TA

Agreement to be accountable for all aspects of the work in ensuring that questions related to the accuracy or integrity of any part of the work are appropriately investigated and resolved; final approval of the version to be published

\section{REFERENCES}

1. Prakash P, Patni R, Asghar NM, Chan KMJ, Antanas M. Ascending aorta aneurysms: pathophysiology and indications of surgery. EJ Cardiol Pract. 2011;10(7):[screen 4 pages].

2. Bentall $H$, De Bono A. A technique for complete replacement of the ascending aorta. Thorax. 1968;23(4):338-9. doi:10.1136/thx.23.4.338.

3. Atik FA, Navia JL, Svensson LG, Vega PR, Feng J, Brizzio ME, et al. Surgical treatment of pseudoaneurysm of the thoracic aorta. JThorac Cardiovasc Surg. 2006;132(2):379-85. doi:10.1016/j.jtcvs.2006.03.052.

4. Niederhäuser U, Künzli A, Genoni M, Vogt P, Lachat M, Turina M. Composite graft replacement of the aortic root: long-term results, incidence of reoperations. Thorac Cardiovasc Surg. 1999;47(5):317-21. doi:10.1055/s-2007-1013165.

5. Mulder EJ, van Bockel JH, Maas J, van den Akker PJ, Hermans J. Morbidity and mortality of reconstructive surgery of noninfected false aneurysms detected long after aortic prosthetic reconstruction. Arch Surg. 1998;133(1):45-9. doi:10.1001/archsurg.133.1.45.

6. Yakut C. A new modified Bentall procedure: the flanged technique. Ann Thorac Surg. 2001;71(6):2050-2. doi:10.1016/s0003-4975(01)02439-0.

7. Oh KT, Derose J, Taub C. Fortune or misfortune: asymptomatic, delayed presentation of complete dehiscence of mechanical aortic valve conduit and pseudoaneurysm. BMJ Case Rep. 2016;2016:bcr2016216320. doi:10.1136/bcr-2016-216320.

8. Kannan A, Smith C, Subramanian S, Janardhanan R. A rare case of prosthetic endocarditis and dehiscence in a mechanical valved conduit. BMJ Case Rep. 2014;2014:bcr2013200720. doi:10.1136/bcr-2013-200720.

9. Stiver K, Bayram M, Orsinelli D. Aortic root bentall graft disarticulation following repair of type a aortic dissection. Echocardiography. 2010;27(2):E27-9. doi:10.1111/j.1540-8175.2009.01069.x.

10. Tabakci MM, Yazicioglu MV, Toprak C, Demirel M, Avci A. Collapse of aortic graft through its disarticulation secondary to periaortic root abscess: an unusual cause of syncope. Echocardiography. 2016;33(8):1267-8. doi:10.1111/echo.13265.

11. Gebhard C, Biaggi P, Stähli BE, Schwarz U, Felix C, Falk V. Complete graft dehiscence 8 months after repair of acute type $A$ aortic dissection. Eur Heart J Acute Cardiovasc Care. 2013;2(1):72-6. doi:10.1177/2048872612471214.

12. Mohammadi S, Bonnet N, Leprince P, Kolsi M, Rama A, Pavie A, et al. 
Reoperation for false aneurysm of the ascending aorta after its prosthetic replacement: surgical strategy. Ann Thorac Surg. 2005;79(1):147-52; discussion 152. doi:10.1016/j.athoracsur.2004.06.032.

13. Brewer RJ, Deck JD, Capati B, Nolan SP. The dynamic aortic root. Its role in aortic valve function. J Thorac Cardiovasc Surg. 1976;72(3):413-7.
14. Thubrikar M, Bosher LP, Nolan SP. The mechanism of opening of the aortic valve. J Thorac Cardiovasc Surg. 1979;77(6):863-70.

15. Lansac E, Lim HS, Shomura Y, Lim KH, Rice NT, Goetz W, et al. A fourdimensional study of the aortic root dynamics. Eur J Cardiothorac Surg. 2002;22(4):497-503. doi:10.1016/s1010-7940(02)00405-0. 